\title{
Revisión sobre la estructura, funcionamiento e histología del sistema cardiovascular de los crustáceos penaeidos.
}

\section{Review of structure, function, and histology of the penaeid crustacean cardiovascular system}

\section{Revisão da estrutura, função e histologia do sistema cardiovascular dos crustáceos penaeídeos}

\author{
Alexander Varela Mejías ${ }^{1}$, Camilo Pohlenz ${ }^{2}$ \\ 1 Laboratorio Sea Farmers, Sinaloa, México. alexander.varela@gmail.com \\ 2 Biomar AS, Global R\&D, Trondheim, Norway. capoh@biomar.com
}

Recibido: 10 de junio de 2020 Corregido: 13 de noviembre de 2020 Aceptado: 20 de noviembre de 2020

\begin{abstract}
Resumen
Las diferencias entre el sistema cardiovascular de los crustáceos decápodos, enfatizando en los Penaeidos, con respecto a los vertebrados y sus implicaciones, han generado un creciente interés en su estudio y comprensión, dada sus actividades inmunoefectoras y fisiológicas. Se presenta el proceso global del recorrido hemolinfático, acompañado de descripciones histológicas de los diferentes órganos, así como los detalles de sus estructuras en especímenes juveniles y adultos de Penaeus vannamei sanos, en secciones histológicas de tejidos procesados mediante técnicas de rutina. Se incluye la descripción del corazón, arterias, órgano linfoide, branquias, lagunas y senos hemales. También, se incluye una reseña, no exhaustiva, sobre los mecanismos de funcionamiento y control asociados, sustentados por información bibliográfica sobre estudios realizados bajo condiciones normales de salud de los camarones examinados. Se incluye valores promedio de ritmo cardíaco a través de los diferentes estadíos de desarrollo, así como valores de volúmenes y presiones arteriales.
\end{abstract}

Palabras clave: sistema cardiovascular, Penaeus, órganos, descripción, corazón, hemolinfa, valores

\begin{abstract}
Studying and understanding the differences between the cardiovascular system of decapod crustaceans, particularly Penaeids, and of vertebrates and their implications has gained growing interest given their immune function and physiological activities. The overall hemolymph path is presented here, along with histological descriptions of the different organs, as well as the details of their structures in healthy juvenile and adult specimens of Penaeus vannamei, in sections of tissues processed using routine techniques. In addition, the heart, arteries, lymphoid organ, gills, lacunas, and hemal sinuses are described. A non-exhaustive review of associated operating and control mechanisms is also included, based on information obtained through bibliographic consultations on studies conducted under normal conditions. Average heart rate values are included in the different stages of development, as well as values of arterial volume and pressure.
\end{abstract}

Keywords: cardiovascular system, Penaeus, organs, description, hearth, hemolymph, values

\section{Resumo}

As diferenças entre o sistema cardiovascular dos crustáceos decápodes, com destaque nos Penaeídeos, no que diz respeito aos vertebrados e suas implicações, têm gerado um interesse crescente no seu estudo e compreensão, dada a sua atividade imunoefetora e fisiológica. O processo global da via hemolinfática é apresentado, 


\begin{abstract}
acompanhado de descrições histológicas dos diferentes órgãos, bem como detalhes de suas estruturas em espécimes de Penaeus vannamei, juvenis e adultos saudáveis, em cortes histológicos de tecidos processados por técnicas de rotina. A descrição do coração, artérias, órgão linfóide, brânquias, lacunas e seios hemais está incluída. Além disso, é incluída uma revisão, não exaustiva, dos mecanismos de operação e controle associados, com base em informações obtidas por meio de consultas bibliográficas sobre estudos realizados em condições normais de saúde do camarão examinado. Os valores médios da frequência cardíaca são incluídos nas diferentes fases de desenvolvimento, bem como os valores dos volumes e das pressões arteriais.
\end{abstract}

Palavras-chave: sistema cardiovascular, Penaeus, órgãos, descrição, coração, hemolinfa, valores

\title{
Introducción
}

Una de las múltiples diferencias entre el sistema cardiovascular, presente en los vertebrados, con respecto a los camarones Penaeidos, radica en que éstos últimos desarrollaron un sistema circulatorio típicamente descrito como abierto o parcialmente abierto, lo cual implica que, la irrigación final de los tejidos se da mediante el paso de la hemolinfa a través de los espacios intersticiales de los diferentes órganos y tejidos sin estar limitado a canales propiamente vasculares (Bell \& Lightner 1988; Cooke 1988; Yazawa et al. 1999; Wilkens et al. 2008; Reiberand \& McGaw 2009; Morales-Covarrubias 2013; Morales \& Cuéllar-Anjel 2014).

Esto, sin embargo, no implica la ausencia de estructuras especializadas. Los crustáceos decápodos cuentan con órganos definidos y desarrollados para la oxigenación de los tejidos, distribución de nutrientes y extracción de sustancias de desecho (Göpel \& Wirkner 2020), lo cual es realizado con gran eficiencia. El sistema cardiovascular de estos animales se puede conceptualizar como un conjunto de cuatro componentes principales, a saber: una bomba (el ventrículo o corazón), la hemolinfa, el sistema arterial y una vía de retorno, análoga a un sistema venoso de los vertebrados (Bell \& Lightner 1988; Figueroa-Soto et al. 1997; Guadagnoli et al. 2011; Göpel \& Wirkner 2020).

Dada la importancia comercial de los camarones de cultivo y captura, se ha realizado múltiples estudios sobre este sistema, sus órganos y tejidos, incluyendo aspectos del desarrollo ontogénico y neurohormonal del corazón (Harper \& Reiber 2001; McMahon et al. 2002), su descripción anatómica e histológica (Bell \& Lightner 1988; Yazawa et al. 1999; Guadagnoli et al. 2011; Göpel \& Wirkner 2020), su respuesta ante estímulos externos (Guadagnoli et al. 2011; Ern et al. 2015; Weineck et al. 2018; Wycoff et al. 2018), la estructura de las arterias (Bell \& Lightner 1988; Wilkens et al. 2008), del órgano linfoide (Bell \& Lightner 1988; Duangsuwan et al. 2008; Rusaini \& Owens 2010) y de los senos hemolinfáticos y branquias, como vía para el retorno de la hemolinfa al corazón (Bell \& Lightner 1988; Göpel \& Wirkner 2020).

Además de sus funciones propias de la distribución de oxígeno, nutrientes y hormonas, así como el retiro de metabolitos de desecho, el sistema cardiovascular de los decápodos cumple funciones de defensa, el cual incluye las ejercidas por células fagocíticas fijas y circulantes (hemocitos), estructuras nodulares y moléculas inmunoefectoras (Lightner 1996; Barracco et al. 2014).

En este estudio, se presenta la información colectada de múltiples publicaciones sobre este importante sistema. Además, se incluye imágenes de secciones histológicas descriptivas del sistema cardiovascular de camarones blancos del Pacífico, Penaeus vannamei, los cuales no presentaban lesiones detectables de carácter patológico. 
Rev. Ciencias Veterinarias, Vol. 38, N² 2, [43-57], E-ISSN: 2215-4507, julio-diciembre, 2020

DOI: https://doi.org/10.15359/rcv.38-2.4

URL: http://www.revistas.una.ac.cr/index.php/veterinaria/index

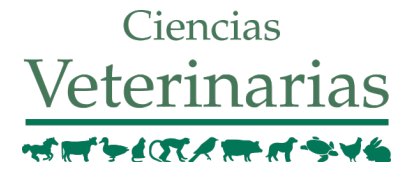

\section{Materiales y Métodos}

Para la obtención del material histológico, se utilizaron especímenes adultos, clínicamente sanos, sin lesiones o patógenos detectados. Los cuales fueron colectados en granjas ubicadas en Sonora, México. Dichos organismos fueron fijados con Davidson AFA, inmediatamente posterior a su captura, para evitar someterlos a estrés adicional y transferirlos a etanol 72 horas después, hasta su procesamiento. Los cortes histológicos se realizaron a $3 \mu \mathrm{m}$ de espesor y se tiñeron con Hematoxilina y Eosina, tal como se describe en Bell \& Lightner (1988). Adicionalmente, la descripción sobre la estructura, funcionamiento e histología del sistema cardiovascular de los crustáceos penaeidos, se sustenta en información obtenida a través de fuentes bibliográficas sobre estudios realizados bajo condiciones normales de salud de los camarones examinados.

\section{El corazón de los decápodos}

La formación del corazón es gradual; ocurre a través del desarrollo larval. En los camarones Metapenaeus ensis, la actividad de bombeo se detecta a partir del estadío de Nauplio 6, con ritmos de 20 a 100 latidos por minuto. Inicialmente, la región contráctil posee dos ingresos para la hemolinfa y la red arterial se limita a un solo vaso anterior. En etapas larvales posteriores al estadio de Misis, se desarrolla un segundo sitio de bombeo posterior, pero conectado con el sitio original. Esta extensión es más muscular, contiene más sitios de ingreso de hemolinfa y desarrolla vasos de distribución adicionales que irrigan el cefalotórax y las áreas abdominales. El sitio original se fusiona gradualmente a la nueva extensión y sólo ocurren pequeños cambios durante las etapas postlarvales y juveniles (McMahon et al. 2002).

Una vez completado su desarrollo, el corazón de los decápodos es una estructura con una única cámara o ventrículo, situado inmediatamente debajo del caparazón en la región dorso posterior del cefalotórax. Este ventrículo está compuesto de músculo estriado, inervado por el ganglio cardíaco situado en la pared dorsal interna del ventrículo (Bell \& Lightner 1988; Yazawa et al. 1999; Göpel \& Wirkner 2020).

Se ubica en la cavidad pericárdica, compuesta de tejido conectivo esponjoso, rodeada por el epicardio y está suspendido por ligamentos originados en la propia cavidad pericárdica. Posee válvulas de ingreso llamadas ostiolos que permiten el paso de la hemolinfa (Bell \& Lightner 1988; Göpel \& Wirkner 2020). El tejido miocárdico es estriado transversal; se separa de la hemolinfa mediante una lámina basal y las células cardiacas se nutren directamente de la hemolinfa (Felgenhauer 1992).

En la cámara pericárdica, se ubican cinco pares de ostiolos por donde ingresa la hemolinfa. Seis ostiolos dorsales y cuatro ventrales. Además, el corazón posee válvulas de salida en las uniones con las arterias que impiden el reflujo de la hemolinfa (Bell \& Lightner 1988; Martin \& Hose 1992; Göpel \& Wirkner 2020).

Según Yazawa et al. (1999), la morfología de las fibras ostiales semeja a la estructura de las fibras miocárdicas; sus haces de filamentos se conectan a las láminas del retículo sarcoplasmático, formando acoplamientos internos entre las bandas sarcoméricas tipo $\mathrm{H}$.

Se considera que el control del ritmo cardíaco es neurogénico, debido a que el miocardio no es activo en forma espontánea (Yazawa et al. 1999). Los impulsos neuronales surgen del ganglio cardíaco, ubicado en el tejido miocárdico (Bell \& Lightner 1988), brindando el pulso sináptico, coordinando las órdenes de cada una de las llamadas células gigantes, las cuales transfieren los impulsos al miocardio. Adicionalmente, se ha demostrado que estas células gigantes influyen directamente sobre las células 
pequeñas, determinando la duración y frecuencia de los latidos (Cooke 1988; Harper \& Reiber 2001). Se ha descrito la acción inhibitoria y aceleradora de los estímulos por un par de nervios que representan las únicas conexiones del ganglio cardíaco con el resto del sistema nervioso. Cada nervio lleva un único inhibidor y dos axones aceleradores (Maynard 1961; Cooke 1988; Reiberand \& McGaw 2009).

Además de la actividad hormonal, se presenta un efecto contráctil resultante de la bomba de intercambio iónico de sodio-potasio. La magnitud de este potencial electroquímico aumenta en función a la concentración del sodio intracelular. Por lo cual, se produce una actividad moderadora del sodio durante las tasas de alta actividad metabólica (Cooke 1988).

Harper \& Reiber (2001), indican que la regulación del ritmo cardíaco en decápodos adultos responde a tres mecanismos de control neurogénico: el ganglio cardíaco, los nervios cardioaceleradores e inhibitorios y las neurohormonas provenientes de los órganos pericárdicos. Así, el funcionamiento cardíaco se basa en la interacción entre el ganglio cardíaco, descrito como un centro celular adecuado para la activación de los mecanismos de espontaneidad, ritmicidad, generación de patrones, integración, la modulación neurohumoral y los nervios asociados. Existe una integración entre impulsos eléctricos y una respuesta hormonal a los sistemas neurosecretores y sus productos, liberados por las estructuras neurohemales (Alexandrowicz \& Carlisle 1953; Cooke 1988; Bell \& Lightner 1988; Harper \& Reiber 2001).

Por su parte, McMahon et al. (2002), indican que el latido cardíaco en M. ensis, en estadios juveniles, se inhibe mediante la inyección de tetrodotoxina. Mientras que, esta respuesta no es observada en estadios larvales tempranos, lo cual sugiere que la regulación neurogénica, a través del ganglio cardíaco, surge más tarde durante el desarrollo.

Adicional al trabajo realizado por el corazón, en algunas especies de crustáceos se ha descrito una estructura de bombeo auxiliar, presente en especies como: el cangrejo Callinectes sapidus, la langosta californiana Panulirus interruptus, el cangrejo de lodo australiano Scylla serrata y en cangrejos marinos como Cancer productus y C. antennarius. Esta estructura se denomina Cor frontale. Se ubica en el extremo anterior de la arteria media dorsal, previo a su ramificación hacia el ganglio supraesofágico y los sistema oculomotor y aparato visual periférico (Steinacker 1978).

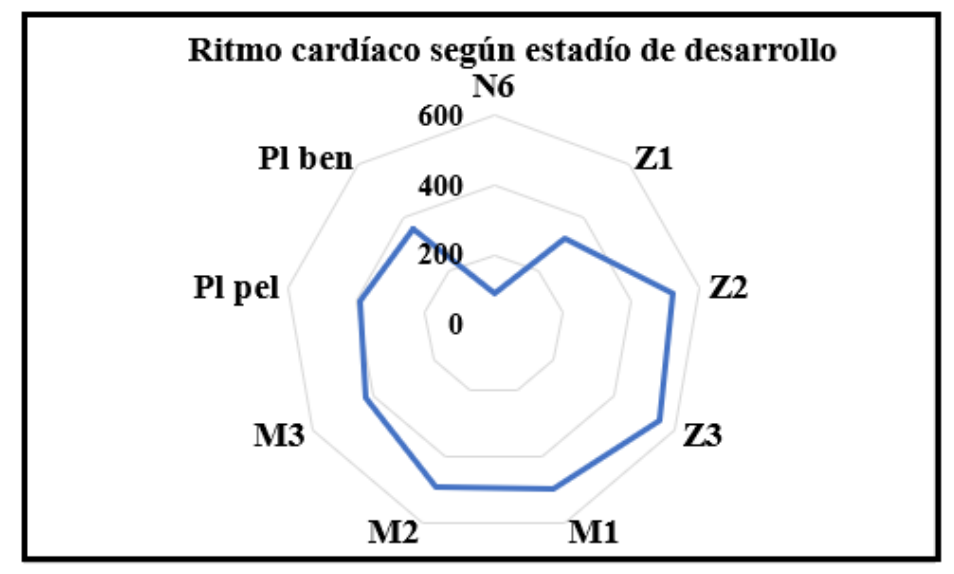

Basado en McMahon et al, 2002 
Las funciones cardíacas responden, entonces, a diferentes reguladores, los cuales actúan por medios hormonales, químicos y nerviosos, variando en función los estímulos internos, entre los cuales se tiene: el estadío de desarrollo, la edad y el tamaño del organismo. Así como a estímulos externos, que actúan como las fuentes de estrés, entre éstos, la temperatura y el oxígeno disuelto (McMahon et al. 2002; Guadagnoli et al. 2011; Aparicio-Simón et al. 2018; Weineck et al. 2018).

Los trabajos realizados por McMahon et al. (2002), en M. ensis, brindaron los siguientes valores promedio de ritmo cardíaco, los cuales fueron medidos en función de los diferentes estadíos de desarrollo de esta especie. En este estudio, se presentan los datos de las determinaciones en los estadios, incluyendo: Nauplio 6, Zoea, Misis, post larva pelágica y postlarva bentónica.

Tal como se observa, el ritmo cardíaco, para esta especie, no es constante, tiene valores medios mínimos en estadío de Nauplio 6, y máximos en Zoea 3.

Similarmente, Guadagnoli et al. (2011), determinaron los siguientes parámetros en el camarón Palaemonetes pugio con un peso promedio de $208.4 \pm 8.9 \mathrm{mg}$, bajo condiciones de salinidad de 30-32 ppt, una temperatura media de $20^{\circ} \mathrm{C}$ y condiciones normales de oxígeno $(20.2 \mathrm{kPa})$.

\begin{tabular}{lc}
\hline \multicolumn{1}{c}{ Parámetro } & Valor promedio \\
\hline Latidos por minuto & 281 \\
$\mu \mathrm{L}$ de hemolinfa por latido & 118.25 \\
Presión sistólica máxima & 22.45 \\
Presión diastólica mínima & -6.85 \\
Volumen de hemolinfa & 33.12 \\
\hline
\end{tabular}

Basado en Guadagnoli et al. 2011

Resulta interesante que la presión diastólica presente valores negativos; lo cual, indicaría una presión menor a la circundante, provocada, según los autores, por la contracción de los ligamentos suspensorios del corazón, dentro de la cámara pericárdica, generando así una fuerza de succión de hemolinfa hacia el ventrículo.

Este valor de ritmo cardíaco es muy cercano al reportado por Weineck et al. (2018), en P. vannamei, con un promedio de 288 latidos por minuto, utilizando animales con un peso promedio de $23.5 \mathrm{~g}$, mantenidos a temperatura con un rango entre 27 y $30.5^{\circ} \mathrm{C}$, con una salinidad de 15 ppt y un oxígeno disuelto de 7.3-7.7 ppm.

Cabe mencionar que los valores expuestos se tomaron de organismos conservados bajo condiciones óptimas. Estos animales, al someterse a estrés, como el generado por niveles bajos de oxígeno o cambios drásticos de temperatura, presentan alteraciones en el control y funcionamiento del sistema cardiovascular, el cual puede modificarse, al generar efectos como: bradicardia, hipotensión arterial, cambios en los volúmenes de hemolinfa impulsados y en la resistencia periférica arterial (McMahon et al. 2002; Guadagnoli et al. 2011; Ern et al. 2015; Aparicio-Simón et al. 2018; Weineck et al. 2018). Estos ajustes fisiológicos tendrían la finalidad de reducir el gasto energético realizado por el corazón, lo cual, a su vez, representa una ventaja para estos animales en casos de hipoxia (Guadagnoli et al. 2011). 
El sometimiento a estrés, por hipoxia, provoca otras modificaciones, incluyendo variaciones en las concentraciones de neurohormonas como: dopamina, octopamina y serotonina; así como cambios en los metabolitos presentes en la hemolinfa. Por ejemplo, durante las hipoxias, se generan reducciones de los niveles de glucosa circulante e incrementos en los niveles de lactato (Aparicio-Simón et al. 2018; Weineck et al. 2018).

Adicionalmente, el corazón de los crustáceos presenta actividades de defensa, con presencia de fagocitos fijos, los cuales tienen la función de capturar patógenos presentes en la hemolinfa. Esto se ha reportado para varias especies, como: Palaemonetes intermedius, P. pugio, Parachaeraps bicarinatus, Homarus americanus, Carcinus maenas y Callinectes sapidus, siendo común encontrar nódulos hemocíticos en casos de presencia bacterial en la hemolinfa (Johnson 1987; Lightner 1996).
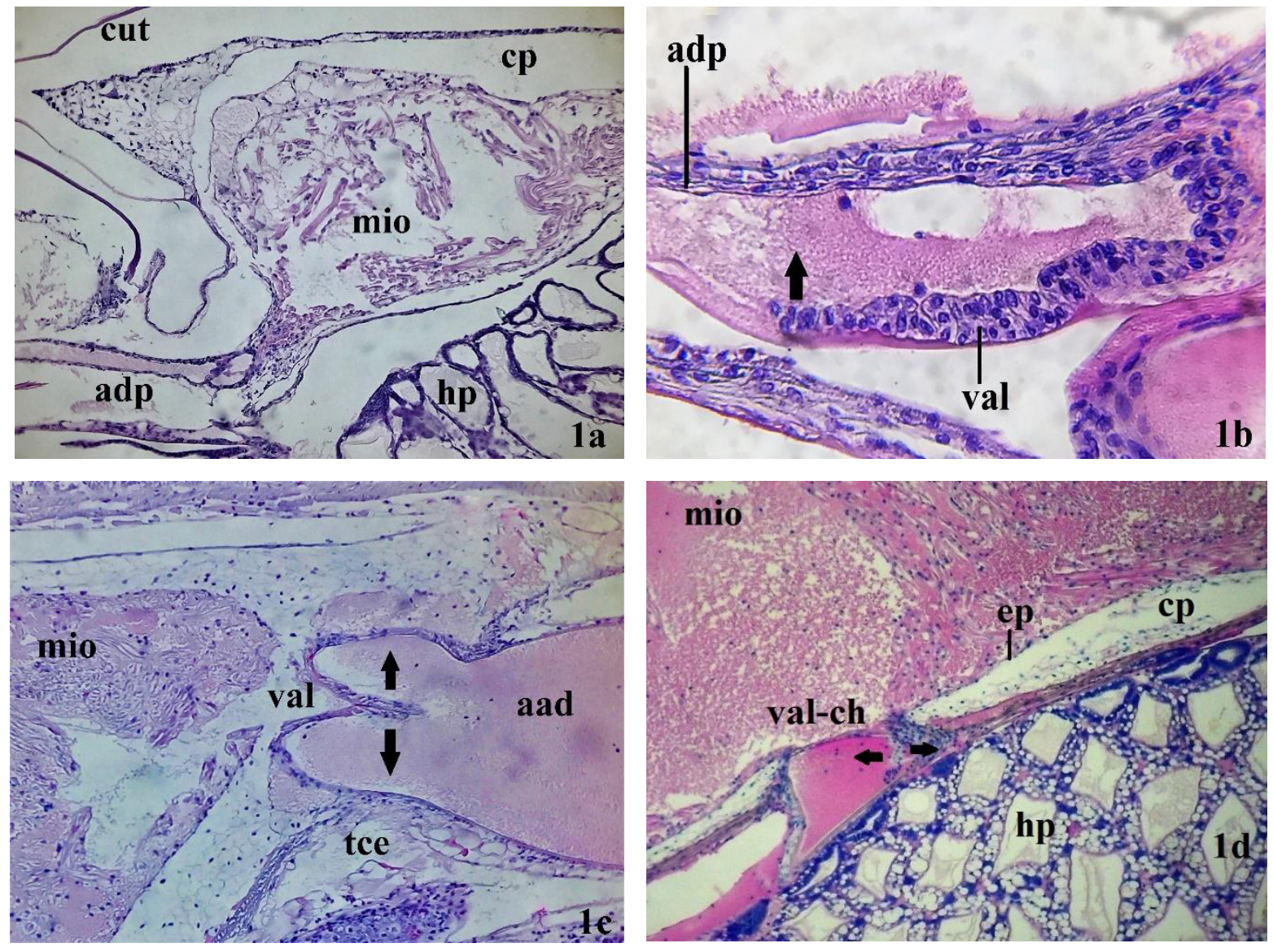

Figura 1. 1a) corte sagital del corazón, se observa la cercanía con la cutícula (cut) en la región dorso anterior del cefalotórax, el tejido miocárdico (mio), conteniendo en la cámara pericárdica (cp), la arteria dorsal posterior (adp) y hepatopáncreas (hp). 1b) Válvula arterial posterior (val), presente en la unión en la salida del corazón hacia la arteria dorso posterior (adp), esta válvula presenta una valva que asciende (en la dirección de la flecha) y permite el paso de la hemolinfa, al descender se impide el reflujo hacia el corazón. 1c) Válvula hacia la arterial anterior dorsal (aad), se señala con marcas la dirección de movimiento de las dos valvas, tejido conectivo esponjoso del pericardio (tce). 1d) Válvula cardio- hepática (val-ch), cerrada en la imagen, se marcan el epicardio (ep), el tejido miocárdico (mio) y parte de la cámara pericárdia. Tinción H\&E. 500 X. 
Rev. Ciencias Veterinarias, Vol. 38, N² 2, [43-57], E-ISSN: 2215-4507, julio-diciembre, 2020

DOI: https://doi.org/10.15359/rcv.38-2.4

URL: http://www.revistas.una.ac.cr/index.php/veterinaria/index

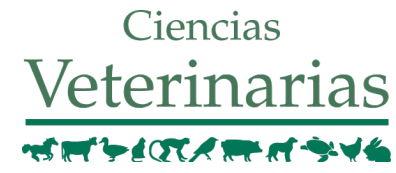

\section{Arterias y venas}

El sistema vascular de los crustáceos inicia en las arterias, las cuales son vasos de pared gruesa. Continúa hacia las arteriolas, más pequeñas con pared fina y los capilares, los cuales son vasos muy pequeños, morfológicamente similares a las arteriolas pero que están relacionados con el intercambio gaseoso y transporte de nutrientes entre la hemolinfa y los tejidos. Así como algunas venas, mediante las cuales circula la hemolinfa oxigenada entre las branquias y el corazón (Bell \& Lightner 1988; Chan et al. 2006; Reiberand \& McGaw 2009; Guadagnoli et al. 2011).

El sistema vascular incluye una serie de arterias, incluyendo a la arteria oftálmica, la cual no es citada por todos los autores, dos arterias laterales, dos arterias hepáticas, una arteria dorsal superior y la arteria descendente. La hemolinfa es distribuida a través del cuerpo de los crustáceos, circulando desde arterias hacia arteriolas y capilares, con un diámetro decreciente. Posteriormente, la hemolinfa abandona los vasos capilares e irriga directamente los tejidos, circulando en los espacios intersticiales hacia los senos hemales o hemolinfáticos (Bell \& Lightner 1988; Martin \& Hose 1992; Guadagnoli et al. 2011). Göpel \& Wirkner (2020), en estudios realizados mediante microtomografías computacionales, moldeo con resinas y microscopía laser confocal, indican que en $P$. vannamei, las arterias anteriores laterales se ramifican y distribuyen hacia regiones anteriores del cuerpo, incluyendo la irrigación oftálmica.

Se ha observado la presencia de válvulas adicionales a las cardíacas, citadas supra. Se trata de válvulas de una sola vía, presentes a lo largo de las arterias principales, estratégicamente ubicadas con el fin de impedir el reflujo de la hemolinfa (Davidson et al. 1998; Wilkens et al. 2008).

Las arterias, que salen del corazón de la langosta, Homarus americanus, han sido descritas como vasos trilaminares que consisten en: una túnica interna, una intermedia y otra externa. Con la túnica interna como un tejido conectivo elástico adyacente al lumen del vaso y la túnica externa siendo un tejido conectivo colágeno (Chan et al. 2006; Wilkens et al. 2008).

La pared de los vasos incluye las siguientes capas, desde el lumen hacia el exterior: íntima, también llamada capa basal o lámina basal, acelular. El endotelio, con una capa celular interna y una capa de tejido conectivo y, finalmente, la lámina externa, también acelular (Bell \& Lightner 1988). Estos autores comentan, además, que se presentan capas celulares adicionales en las regiones iniciales de las arterias: oftálmica, laterales, hepáticas, dorsal superior y arteria descendente, formando agregados celulares compactos. Las capas intermedias de las arterias, se encuentran menos compactadas conforme estas reducen su diámetro, siendo infiltradas por elementos de tejido conectivo (Bell \& Lightner 1988; Wilkens et al. 2008).

En el caso de la arteria dorsal, se ha reportado haces de microfilamento, atravesando el citoplasma; se asocian con frecuencia con redes filamentosas en la superficie interna del plasmalema. Interesantemente, con respecto a la presencia de fibras musculares asociadas a las arterias mayores, dichas fibras tendrían participación activa en el movimiento de la hemolinfa, actuando como sistemas de apoyo para el corazón (Wilkens et al. 2008).

Estas modificaciones por presencia de fibras, consisten en capas internas de tejido conectivo elástico y capas externa de tejido conectivo. En la capa media de las arterias se presentan agregados de células que contienen los microfilamentos citados. Estas células arteriales poseen actina, miosina y tropomiosina. Las células 

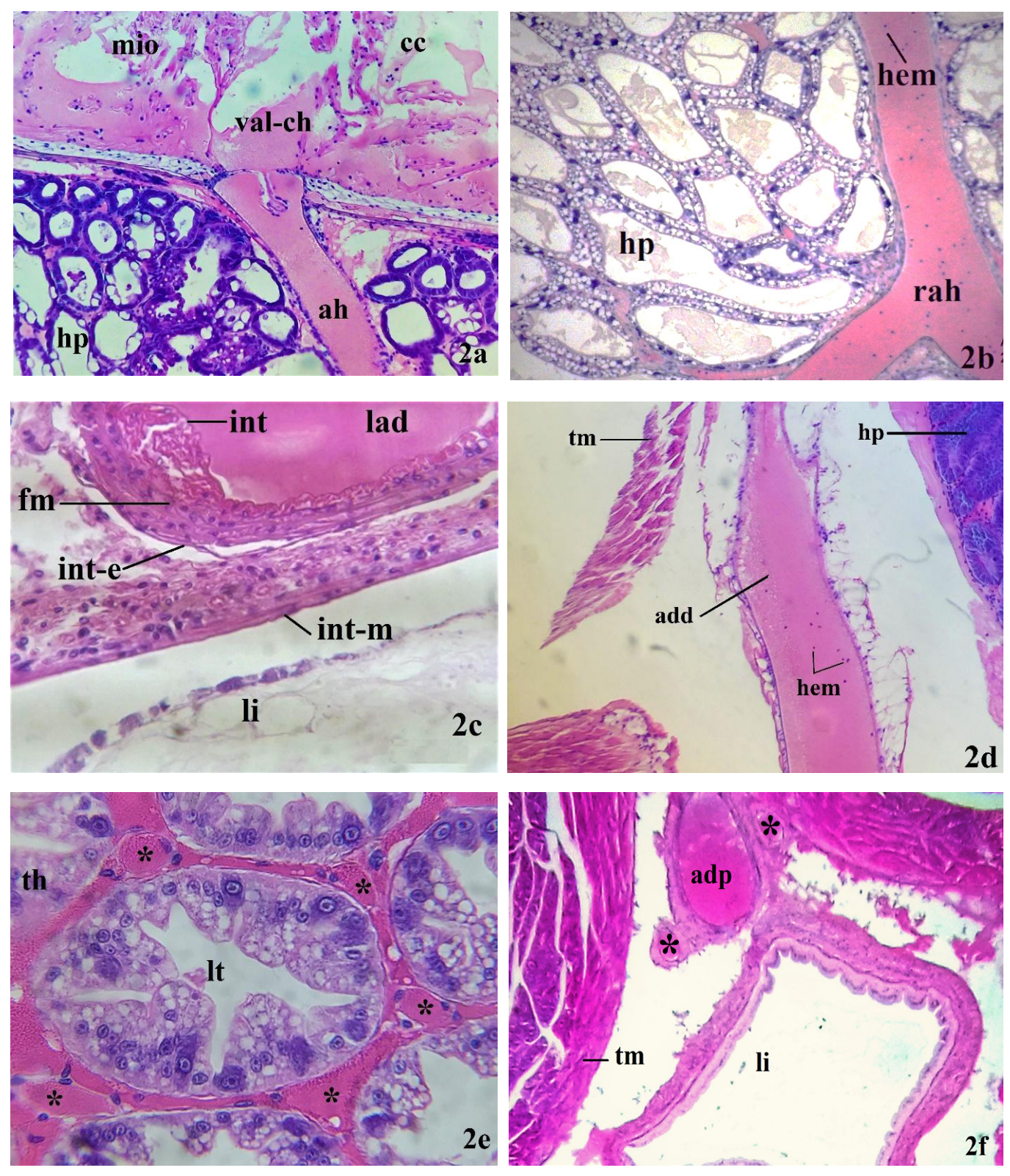

Figura 2. 2a) panorámica de zona de ingreso de la arteria hepática (ah), se observa la válvula cardio hepática cerrada (valch), así como la cámara cardíaca (cc), el tejido miocárdico (mio), y hepatopáncreas (hp). 2b) Corte sagital de hepatopáncreas, ligeramente más ventral con respecto a la imagen anterior. Hepatopáncreas (hp) con cortes en los túbulos en forma oblicua, se presenta una ramificación de la arteria hepática (rah). 2c) Corte transverso, se presenta el lumen de la arteria dorsal posterior (lad), se señala la íntima -acelular-, así como la íntima externa (int-e), las fibras musculares (fm) en la capa intermedia. Además, se observa la membrana basal del intestino medio (int.m) y el lumen intestinal (li). 2d) vista de arteria descendente dorsal (add), posterior al hepatopáncreas (hp). Se observa tejido muscular estriado (tm). En el lumen arterial se observan hemocitos, presentes como estructuras esféricas, basofílicas. 2e) túbulos hepatopancreáticos (th), de la región media tubular. Se observa el lumen tubular (lt), senos hemales entre los espacios intertubulares (asteriscos). 2f) corte transverso en el $4^{\circ}$ segmento abdominal, presencia del lumen del intestino medio (li), tejido muscular ( $\mathrm{tm}$ ). Paralela al intestino, se observa la arteria dorsal posterior (adp) y un par de arteriolas adyacentes (asteriscos). Tinción H\&E. 500 X. 
musculares estriadas típicas no son evidentes en las paredes de ninguno de los vasos. Neurotransmisores, como el ácido glutámico y la neurohormona proctolina, provocarían contracciones circunferenciales lentas en todas las arterias que salen del corazón de la langosta H. americanus (Wilkens et al. 2008); estas fibras han sido reportadas también en otras especies como P. stylirostris (Bell \& Lightner 1988).

La existencia de venas es limitada o inexistente en decápodos, siendo su papel el retornar la hemolinfa oxigenada, desde las branquias hacia el seno pericárdico, evitando la mezcla de hemolinfa oxigenada y no oxigenada (Bell \& Lightner 1988; Martin \& Hose 1992). Göpel \& Wirkner (2020) por su parte, indican que en $P$. vannamei el retorno no se da mediante venas, sino a través de senos branquio-pericárdicos.

\section{Órgano linfoide}

El órgano linfoide consiste en una pequeña estructura bilobulada. Se localiza en la región antero ventral del hepatopáncreas y posterior a la glándula antenal, la cual ha sido reportada únicamente para especies de camarones penaeidos. Entre estos: Penaeus chinensis, P. monodon, P. vannamei. P. stylirostris y $P$. japonicus (Lightner et al. 1987; Bell \& Lightner 1988; Shao et al. 2004; Rusaini \& Owens 2010; Barraco et al. 2014).

Cada lóbulo está conformado por túbulos que poseen diferentes tipos de células en la matriz estromal y senos intersticiales. Los túbulos están rodeados con fibra de tejido conectivo, con senos hemolinfáticos que ocupan los espacios entre los túbulos (Rusaini \& Owens 2010).

El lumen tubular se encuentra revestido, internamente, por células endoteliales aplanadas. Desde este lumen, los hemocitos pueden ingresar a través de la capa de células endoteliales, movilizarse hacia la matriz estromal y penetrar en el sistema circulatorio abierto de los senos hemolinfáticos (Hasson et al. 1999; van de Braak et al. 2002; Duangsuwan et al. 2008; Rusaini \& Owens 2010).
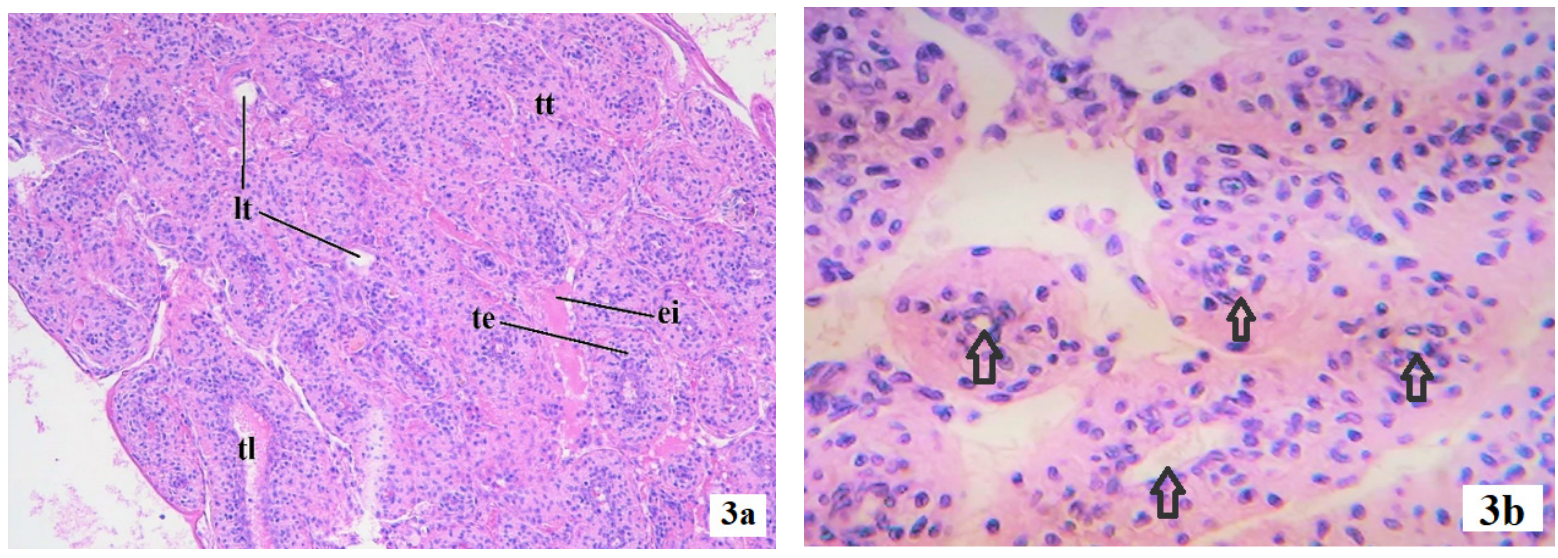

Figura 3a. Órgano linfoide visto en menor magnificación, se indican algunos de los lúmenes tubulares (lt), así como túbulos con cortes longitudinales ( $\mathrm{tl}$ ) y transversales ( $\mathrm{tt}$ ). Se señala una zona con espacios intertubulares o senos intertubulares (ei) y tejido estromático (te) en la pared de uno de los túbulos transversos. 3b: Vista de órgano linfoide, se presenta como agregados celulares con disposición tubular transversal, se muestra lumen central en algunos de ellos (flechas). Tinción H\&E. 200 X y 400 X. 
Al seguir su localización, la arteria subgástrica se conecta directamente al órgano linfoide; se ramifica varias veces formando un plexo vascular dentro del órgano. Finalmente, se da lugar a capilares terminales de pequeño diámetro, en muchas ocasiones ocluidos (Bell \& Lightner 1988; Hasson et al. 1999; van de Braak et al. 2002; Duangsuwan et al. 2008; Rusaini \& Owens 2010; Morales-Covarrubias 2013).

El tamaño del órgano linfoide dependerá del tamaño del organismo de la especie y de su estado sanitario. Se ha reportado rangos de diámetro que van desde los $2 \mathrm{~mm}$, para ejemplares de $P$. chinensis con tamaño corporal de $13 \mathrm{~cm}$., hasta $4 \mathrm{~mm}$ para P. monodon de $20 \mathrm{~cm}$ de talla aproximada (Rusaini \& Owens 2010). En $P$. vannamei el tamaño es de aproximadamente $1.5 \mathrm{~mm}$ para reproductores de $35 \mathrm{~g}$ (observación personal).

Funcionalmente, este órgano es considerado parte integral del sistema inmune de los camarones, participando activamente en el reconocimiento, secuestro, aislamiento y eliminación de agentes extraños (Lightner et al. 1987; Bell \& Lightner 1988; Owens et al. 1991; Bonami et al. 1992; Kondo et al. 1994; Hasson et al. 1999; Shao et al. 2004; Poulos et al. 2006; Duangsuwan et al. 2008; Rusaini \& Owens 2010; Lightner 2011; Zhang et al. 2014; Peña-Navarro \& Varela-Mejías 2015).
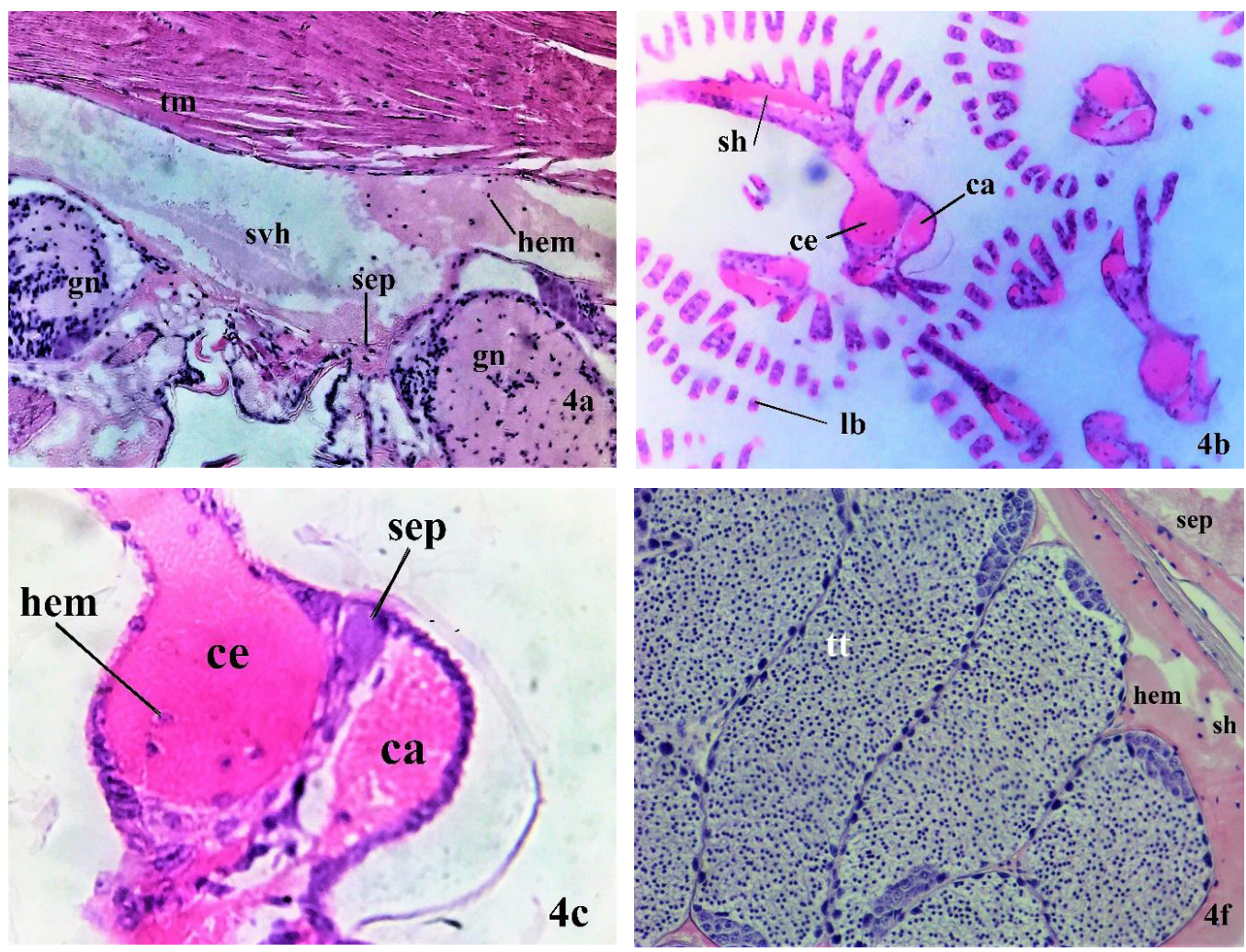

Figura 4. Corte sagital de la región ventral del cefalotórax. Se observa el seno ventral hemal (svh), entre el tejido muscular (tm) y los ganglios nerviosos (gn). Se señala el septum y se observan algunos hemocitos. 4b) Vista general de las lámelas branquiales, señalando el canal eferente (ca) y el canal eferente (ce), en la base de un árbol branquial. Se indican las algunas lámelas branquiales terminales (lb) y el seno hemal (sh) en una lámela. 4c) base ampliada de árbol braquial, se observa con mayor detalle a los canales aferente y eferente, así como el septum (sep) que los separa y algunos hemocitos presentes. 4f) Testis de reproductor, en la periferia se observa el septum y el seno hemolinfático (sh) y hemocitos presentes en la hemolinfa (hem). Tinción H\&E. 100 X y 400 X 
Rev. Ciencias Veterinarias, Vol. 38, N 2, [43-57], E-ISSN: 2215-4507, julio-diciembre, 2020

DOI: https://doi.org/10.15359/rcv.38-2.4

URL: http://www.revistas.una.ac.cr/index.php/veterinaria/index

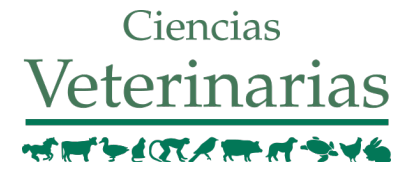

\section{Lagunas y Senos hemales}

Después de abandonar las arterias, arteriolas y capilares, la hemolinfa fluye a través de los diferentes tejidos y órganos, dirigiéndose hacia las lagunas y senos hemolinfáticos, proceso en el cual tiene lugar el intercambio de nutrientes y gases. (Sis et al. s.f; Bell \& Lightner 1988).

Las lagunas y senos hemales constituyen áreas en las cuales se almacena y transporta la hemolinfa, presentando diferencias estructurales entre ellos. Los senos están, al menos parcialmente, bordeados por paredes de tejido conectivo; en tanto que las lagunas no presentan tales paredes, sino que se constituyen de los espacios presentes entre órganos (Göpel, T. Comunicación personal).

Estas lagunas y senos se distribuyen en diferentes regiones anatómicas, por ejemplo, la laguna perineural rodea al cordón nervioso y los músculos estriados torácicos. Se trata de áreas de acumulación de hemolinfa, formando una red de lagunas con una morfología similar a los capilares, con la diferencia de no poseer un verdadero revestimiento endotelial y presentar mayor volumen (Reiberand \& McGaw 2009). Su función principal es la de canalizar la hemolinfa a través de los órganos, permitiendo el intercambio de nutrientes y oxígeno, a la vez que acarrea metabolitos de desecho.

\section{Branquias de los camarones penaeidos}

Las branquias presentan un axis central con numerosas láminas secundarias, las cuales dan lugar a filamentos, divididos en dos ramas cerca de sus extremos. El eje central está unido a la pared cefalotorácica a través de una estructura tubular. Los filamentos primarios se ramifican desde el eje central; cada filamento primario se divide aún más en filamentos secundarios. El tabique longitudinal divide la luz de cada eje, rama y filamento en vasos aferentes y eferentes, separados por un septum, el cual evita que se mezclen hemolinfa oxigenada y no oxigenada. La superficie de cada lámina o filamento está cubierta por una cutícula delgada debajo de una monocapa epitelial. Cada punta de filamento tiene un seno hemolinfático. La hemolinfa del seno esternal fluye a través de los canales aferentes hacia los filamentos, donde se dirige a los canales eferentes (Bell \& Lightner 1988; Figueroa-Soto et al. 1997; Wu et al. 2009). La hemolinfa, finalmente, retorna a la cavidad pericárdica a través del seno branquio-pericárdico (Göpel \& Wirkner 2020).

Además de estos canales, numerosos vasos sanguíneos impregnan las branquias. La cutícula, que cubre las branquias, se superpone a un epitelio delgado que está separado de la hemolinfa por una lámina basal. El epitelio tiene una serie de procesos pilares que forman lagunas subcuticulares. Los nefrocitos, que se asemejan a los podocitos glomerulares, recubren los canales eferentes (Foster \& Howse 1978; Bell \& Lightner 1988).

Göpel \& Wirkner (2020), plantean un modelo mediante el cual la hemolinfa se expele del corazón a través de las arterias y sus ramificaciones, abandona las arterias y entra al sistema lacunar, fluyendo alrededor del intestino medio y hepatopáncreas, mediante varias lagunas interconectadas. Aquí, es probable que los nutrientes se absorban en la hemolinfa, a la vez que el hepatopáncreas reciba oxígeno.

Según este modelo, la hemolinfa se acumula en la laguna ventral desde donde se canaliza hacia la laguna perineural mediante la conexión entre las dos lagunas o a través de pequeñas lagunas en los intersticios de la musculatura ventral. De este modo, la hemolinfa puede suministrar, al cordón nervioso ventral, los nutrientes colectados en el hepatopáncreas, antes de entrar en las lagunas ventrolaterales que emergen desde la laguna perineural. Posteriormente, la hemolinfa ingresa a las lagunas branquiales 
aferentes para pasar hacia las ramas terminales de las branquias, en las cuales se encuentra pequeñas lagunas, dando lugar al intercambio de gases. La hemolinfa oxigenada, aún portadora de nutrientes, fluye de regreso al cefalotórax a través de las lagunas branquiales eferentes, las cuales se abren en los senos branquio-pericárdicos; la hemolinfa reingresa al seno pericárdico, desde donde puede volver a entrar al corazón a través de los ostiolos (Göpel \& Wirkner 2020).

\section{Discusión y conclusiones generales}

Los crustáceos decápodos poseen sistemas cardiovasculares estructurados, diseñados para permitir el impulso de la hemolinfa, desde el corazón hacia los diferentes órganos y tejidos. Para esto dispone de sistemas valvulares e impulsores que impiden el reflujo de la hemolinfa hacia el corazón, sin antes haber recorrido el cuerpo del animal.

El proceso de bombeo de hemolinfa se da como resultado de la acción mecánica del corazón, apoyado por acciones adicionales. Por ejemplo, la presencia de estructuras auxiliares de rebombeo en algunas especies y la presencia de fibras musculares en algunos de los principales vasos que impulsan a la hemolinfa.

El funcionamiento del sistema cardiovascular responde a estímulos ganglionares, electroquímicos y hormonales. Estos, a su vez, reaccionan a las condiciones en la cuales se encuentran los animales; también, a su estado de desarrollo. Ante condiciones adversas, los crustáceos tratarán de ajustar su metabolismo, con el fin de lograr menor inversión de energía en los procesos basales, al ser energéticamente favorable para ellos.

Se ha determinado los valores de normalidad para varias especies de crustáceos, incluyendo la presión arterial, ritmo cardíaco y volúmenes de hemolinfa movilizados. Estos mantienen, bajo condiciones de normalidad, valores estables y se ajustan en casos de condiciones de estrés, al generar respuestas que incluyen bradicardia, reducción de los volúmenes de hemolinfa movilizada, ajustes en los pigmentos respiratorios y modificaciones en la concentración de hormonas neuroefectoras.

Además de las funciones propias de la oxigenación, el sistema cardiovascular de los decápodos incluye funciones de defensa, entre ellas la presencia de células con capacidad fagocítica, así como el reconocimiento, aislamiento y neutralización de algunos patógenos.

El modelo presentado por Göpel \& Wirkner (2020) tiene la finalidad de facilitar la comprensión de las relaciones funcionales entre los senos y las lagunas en términos de suministro de nutrientes y oxígeno a los tejidos y órganos. Sin embargo, los mismos autores indican que es evidente que los procesos descritos suceden simultáneamente y que la hemolinfa enriquecida con nutrientes y la hemolinfa oxigenada, no pueden separarse tan estrictamente como podría implicar el modelo cuando se interpreta literalmente. Sin embargo, también es obvia la importancia funcional del sistema lacunar.

Las lagunas y senos hemales participan, activamente, en el suministro de oxígeno y nutrientes a los diversos órganos. El cordón nervioso ventral, por ejemplo, es alimentado por la hemolinfa en la laguna perineural. El intercambio de gases branquiales se lleva a cabo en las lagunas branquiales; la absorción de nutrientes ocurre en pequeñas lagunas alrededor del intestino medio y del hepatopáncreas (Göpel \& Wirkner 2020). Esto confiere mayor importancia a estas regiones, dado que previamente eran consideradas simples áreas de acumulación de hemolinfa previo a su retorno al corazón.

La concepción de sistema abierto, como concepto general, ha sido utilizada desde hace mucho tiempo con la finalidad de distinguir éstos de los sistemas "normales" o "típicos", los cuales se consideran cerrados, tal como los sistemas de los vertebrados. Sin embargo, los conocimientos actuales hacen dudar 
Rev. Ciencias Veterinarias, Vol. 38, No 2, [43-57], E-ISSN: 2215-4507, julio-diciembre, 2020

DOI: https://doi.org/10.15359/rcv.38-2.4

URL: http://www.revistas.una.ac.cr/index.php/veterinaria/index

Ciencias

Veterinarias

de este concepto rígido de sistema abierto, para dar lugar a un modelo en el cual se dispone de rutas bien establecidas de salida, distribución y retorno de la hemolinfa a través de los diferentes órganos y sistemas. Los sistemas de lagunas y senos dejan de ser simples lugares de acumulación para pasar a ser partes fundamentales con funciones de intercambio de sustancias, durante la circulación de la hemolinfa.

Conflicto de intereses: Los autores declaramos que no poseemos conflicto de intereses con los temas expuestos.

\section{Referencias}

Alexandrowicz, J. S., and Carlisle, D. B. 1953. Some experiments on the function of the pericardial organs in Crustacea. J. mar. biol. Ass. U. K. 32 (1953) 175-192.

Aparicio-Simón, B., Piñón, M., Racotta, R. \& Racotta, I.S. 2018. Neuroendocrine and metabolic responses of Pacific whiteleg shrimp Penaeus vannamei exposed to hypoxia stress. Lat. Am. J. Aquat. Res. 46(2): 364-376. doi: 10.3856/vol46-issue2-fulltext-12

Barracco, M.A., Perazzolo, L.M. \& Rosa, R.D. 2014. Avances en la Inmunología del Camarón. In: Morales, V. \& Cuéllar-Anjel, J. (Eds.). Guía Técnica - Patología e Inmunología de Camarones Penaeidos. OIRSA, Panamá, Rep. de Panamá, p. 237-306.

Bell, T.A. \& Lightner, D.V. 1988. A handbook of normal penaeid shrimp histology. The World Aquaculture Society, Baton Rouge, L.A.

Bonami, J.R., Lightner, D.V., Redman, R.M. \& Poulos, B.T. 1992. Partial characterization of a togavirus (LOVV) associated with histopathological changes of the lymphoid organ of penaeid shrimps. Dis. Aquat. Organ. 14: 145-152.

Chan, K.S., Cavey, M.J. \& Wilkens, J.L. 2006. Microscopic anatomy of the thinwalled vessels leaving the heart of the lobster Homarus americanus: anterior lateral arteries. Invertebr. Biol. 125(1): 70-82. doi: 10.1111/j.1744-7410.2006.00042.x

Cooke, I.M. 1988. Studies on the crustacean cardiac ganglion. Comp. Biochem. Phys. C. 91(1): 205-218. doi: 10.1016/0742-8413(88)90188-0

Davidson G.W., Wilkens J.L., Lovell P. 1998. Neural Control of the Lateral Abdominal Arterial Valves in the Lobster Homarus americanus. Biol Bull. 194(1):72-82. doi: 10.2307/1542515

Duangsuwan, P., Phoungpetchara, I., Tinikul, Y., Poljaroen, J., Wanichanon, C. \& Sobhon, P. 2008. Histological and three-dimensional organizations of lymphoid tubules in normal lymphoid organ of Penaeus monodon. Fish Shellfish Immun. 24(4): 426-435. doi: 10.1016/j.fsi.2007.12.011

Ern, R., Thanh Huong, D.T., Phuong, N.T., Teglberg, P., Wang, T. \& Bayley, M. 2015. Some like it hot: Thermal tolerance and oxygen supply capacity in two eurythermal crustaceans. Sci. Rep. 5(10743): 1-11. doi: 10.1038/srep10743.

Felgenhauer, B.E. 1992. Internal anatomy of Decapoda: An overview. In: Harrison F.W. \& Humes, A.G. (Eds.). Microscopic anatomy of invertebrates. Decapoda Crustacea. Wiley-Liss Inc., Nueva York, p. 45-75.

Figueroa-Soto, C.G., Calderón de la Barca, A.M., Vázquez-Moreno, L., Higuera-Ciapara, I. \& YepizPlascencia, G. 1997. Purification of hemocyanin from white shrimp (Penaeus vannamei Boone 1931) by immobilized metal affinity chromatography. Comp. Biochem. Physiol. 117(2): 203-208. 
Foster, C.A. \& Howse, H.D. 1978. A morphological study on gills of the brown shrimp, Penaeus aztecus. Tissue Cell 10(1): 77-92. doi: 10.1016/0040-8166(78)90008-3

Göpel, T. \& Wirkner, C.S. 2020. The circulatory system of Penaeus vannamei Boone, 1931 - Lacunar function and a reconsideration of the "open vs. closed system" debate. J. Morphol. 281(4-5): 500512. doi: 10.1002/jmor.21117

Guadagnoli, J.A., Tobita, K. \& Reiber, C.L. 2011. Changes in cardiac performance during hypoxic exposure in the grass shrimp Palaemonetes pugio. J. Exp. Biol. 214: 3906-3914. doi: 10.1242/jeb.060442

Hasson, K., Lightner, D.V., Mohney, L., Redman, R. \& White, B. 1999. Role of lymphoid organ spheroids in chronic Taura syndrome virus (TSV) infections in Penaeus vannamei. Dis. Aquat. Organ. 38(2): 93-105. doi: $\underline{10.3354 / \text { dao038093 }}$

Harper, S.L. \& Reiber, C.L. 2001. Ontogeny of neurohormonal regulation of the cardiovascular system in crayfish Procambarus clarkii. J. Comp. Physiol. B 171: 577-583. doi: 10.1007/s003600100208

Johnson, P.T. 1987. A review of fixed phagocytic and pinocytotic cells of decapod crustaceans, with remarks on hemocytes. Dev. Comp. Immunol. 11(4): 679-704. doi: 10.1016/0145-305x(87)90057-7

Kondo, M., Itami, T., Takahashi, Y., Fujii, R. \& Tomonaga, S. 1994. Structure and function of the lymphoid organ in the Kuruma prawn. Dev. Comp. Immunol. 18: S109.

Lightner, D.V. 1996. A handbook of shrimp pathology and diagnostic procedures for diseases of cultured penaeid shrimp. World Aquaculture Society, Baton Rouge, L.A., USA.

Lightner, D, V. 2011. Virus diseases of farmed shrimp in the Western Hemisphere (the Americas): A review. J. Invertebr. Pathol. 106(1): 110-130. doi: 10.1016/j.jip.2010.09.012

Lightner, D.V., Hedrick, R.P., Fryer, J.L., Chen, S.N., Liao, I.C. \& Kou, G.H. 1987. A survey of cultured penaeid shrimp in Taiwan for viral and other important diseases. Fish Pathol. 22(3): 127-140. doi: $10.3147 /$ jsfp. 22.127

Martin, G.G. \& Hose, J.E. 1992. Vascular elements and blood (hemolymph). In: Harrison, F.W. \& Humes, A.G. (Eds.). Microscopic anatomy of invertebrates. Decapod Crustacea. Wiley-Liss, Inc., New York, p.117-146.

Maynard, D. M. 1961. Cardiac inhibition in decapod Crustacea, in: Nervous inhibition, pp. 144-178. Ed. E. Florey. Pergamon Press, New York.

McMahon, B.R., Tanaka, K., Doyle, J.E. \& Chu, K.H. 2002. A change of heart: cardiovascular development in the shrimp Metapenaeus ensis. Comp. Biochem. Phys. A 133(3): 577-587. doi: $\underline{10.1016 / S 1095-6433(02) 00196-4}$

Morales-Covarrubias, M.S. 2013. Camarón Análisis en Fresco, herramienta de diagnóstico. CIAD-OIRSA, p.86.

Morales, V. \& Cuéllar-Anjel, J. 2014. Guía Técnica - Patología e Inmunología de Camarones Penaeidos. OIRSA, Panamá, Rep. de Panamá, 382pp.

Owens, L., De Beer, S. \& Smith, J. 1991. Lymphoidal parvovirus-like particles in Australian penaeid prawns. Dis. Aquat. Organ. 11: 129-134.

Peña-Navarro, N. \& Varela-Mejías, A. 2015. Análisis histopatológico en Litopenaeus vannamei infectado con Vibrio parahaemolyticus. Agron. Mesoam. 26(1):43-53. doi 10.15517/am.v26i1.16892 
Rev. Ciencias Veterinarias, Vol. 38, No 2, [43-57], E-ISSN: 2215-4507, julio-diciembre, 2020

DOI: https://doi.org/10.15359/rcv.38-2.4

Ciencias

URL: http://www.revistas.una.ac.cr/index.php/veterinaria/index

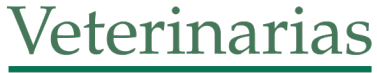

Poulos, B.T., Tang, K.F.J., Pantoja, C.R., Bonami, J.R. \& Lightner, D.V. 2006. Purification and characterization of myonecrosis virus of penaeid shrimp. J. Gen. Virol. 87(4): 987-996. doi: 10.1099/vir.0.81127-0

Reiberand, C.L. \& McGaw, I.J. 2009. A Review of the "Open" and "Closed" Circulatory Systems: New Terminology for Complex Invertebrate Circulatory Systems in Light of Current Findings. Int. J. Zool. 2009: 1-8. doi: 10.1155/2009/301284

Rusaini \& Owens, L. 2010. Insight into the lymphoid organ of penaeid prawns: A Review. Fish Shellfish Immun. 29 (3): 367-377. doi: 10.1016/j.fsi.2010.05.011

Shao, M.Y., Zhang, Z.F., Kang, K.H., Chen, Z.T. \& Kim, J.M. 2004. The study on the cytology and histochemistry of lymphoid organ spheroids in Penaeus chinensis. Aquaculture 240(1-4): 463471. doi: 10.1016/j.aquaculture.2004.02.027

Sis, R.F., Lewis, D.H. \& Means, J.E. (s. f) Shrimp: An Anatomical Study. IAAAM Archive.

Steinacker, A. 1978. The anatomy of the decapod crustacean auxiliary heart. Biol. Bull. 154(3): 497-507. doi: $10.2307 / 1541075$

van de Braak, C.B.T., Botterblom, M.H.A., Taverne, N., van Muiswinkel, W.B., Rombout, J.H.W.M. \& van der Knaap, W.P.W. 2002. The roles of haemocytes and the lymphoid organ in the clearance of injected Vibrio bacteria in Penaeus monodon Shrimp. Fish Shellfish Immun. 13(4): 293-309. doi: $\underline{10.1006 / \text { fsim.2002.0409 }}$

Weineck, K., Ray, A.J., Fleckenstein, L.J., Medley, M., Dzubuk, N., Piana, E. \& Cooper, R.L. 2018. Physiological Changes as a Measure of Crustacean Welfare under Different Standardized Stunning Techniques: Cooling and Electroshock. Animals 8(9): 158. doi: 10.3390/ani8090158

Wilkens, J.L., Cavey, M.J., Shovkivska, I., Zhang, M.L. \& ter Keurs, H.D.E.J. 2008. Elasticity, unexpected contractility and the identification of actin and myosin in lobster arteries. J. Exp. Biol. 211: 766772. doi: $10.1242 /$ jeb. 007658

Wycoff, S., Weineck, K., Conlin, S., Suryadevara, C., Grau, E., Bradley, A., Cantrell, D., Eversole, S., Grachen, C., Hall, K., Hawthorne, D., Kinmon, K., Ortiz, P., Patel, B., Samuels, K., Valdes, K., Ray, A., Fleckenstein, L., Piana, E. \& Cooper, R. 2018. Effects of clove oil (eugenol) on proprioceptive neurons, heart rate, and behavior in model crustaceans. Impulse: The Premier Journal for Undergraduate Publications in the Neurosciences.

Wu, J.P., Chen, H.C. \& Huang, D.J. 2009. Histopathological alterations in gills of white shrimp, Litopenaeus vannamei (Boone) after acute exposure to cadmium and zinc. B. Environ. Contam. Tox. 82(90): 90-95. doi: 10.1007/s00128-008-9582-7

Yazawa, T., Wilkens, J.L., ter Keurs, H.E.D.J. \& Cavey, M.J. 1999. Structure and contractile properties of the ostial muscle (musculus orbicularis ostii) in the heart of the American lobster. J. Comp. Physiol. B 169: 529-537. doi: 10.1007/s003600050252

Zhang, Q., Liu, Q., Liu, S., Yang, H., Liu, S., Zhu, L., Yang, B., Jin, J., Ding, L., Wang, X., Liang, Y., Wang, Q. \& Huang, J. 2014. A new nodavirus is associated with covert mortality disease of shrimp. J. Gen. Virol. 95(12): 2700-2709. doi: 10.1099/vir.0.070078-0 